\title{
On the Error Exponent of Trellis Source Coding
}

\author{
Ilan Hen and Neri Merhav \\ Department of Electrical Engineering \\ Technion - Israel Institute of Technology \\ Haifa 32000, ISRAEL \\ \{ilanh@tx,merhav@ee\}.technion.ac.il
}

\begin{abstract}
In this paper, we develop a single-letter lower bound on the error exponent for the problem of trellis source coding. We demonstrate that for the case of a binary source with the Hamming distortion measure, and for rates close to the rate-distortion curve, this bound is superior to Marton's block coding exponent, for the same computational complexity.
\end{abstract}

Index Terms - Block source coding, block source coding error exponent, computational complexity, rate-distortion function, relative entropy, trellis source coding, trellis source coding error exponent, Viterbi algorithm.

\section{Introduction}

Historically, trellis source codes were developed in analogy to trellis channel codes (specifically, convolutional codes), whose performance/complexity tradeoff was shown to be better than that of block channel codes [22]. Trellis channel codes also serve as building blocks for nowadays state-of-the-art channel codes, like Turbo codes. Thus, it should not be surprising that trellis source codes are superior to block source codes in terms of having better performance/complexity tradeoff, as demonstrated empirically, e.g., [5], [6], [9], [14], [15], [24], [25], [26], and analytically [21], [29].

Jelinek [13] was the first to conjecture that trellis source codes are asymptotically opti- 
mal, i.e., the code performance asymptotically achieves the rate-distortion function as the constraint length grows without bound. Omura [17] also demonstrated that optimal trellis source encoding can be done by using the Viterbi algorithm [20]. Viterbi and Omura [21], have proven the Jelinek conjecture to be true. They have proven a trellis source coding theorem for memoryless sources. Using a random coding argument, they proved that there exists a time-varying trellis code that is asymptotically optimal. They also provided the rate at which the code performance converges to the rate-distortion function, showing it to be better than the one corresponding to block source codes. Gray [8], extended the trellis source coding theorem to stationary and ergodic sources. Using various theorems on sliding-block source codes [7], [10], [11], he proved that there exists a time-invariant trellis code that is asymptotically optimal. However, since he considered general ergodic sources, he could not provide an estimate on the convergence rate of the distortion towards the distortion-rate function. The theorem of Viterbi and Omura and the theorem of Gray are both existence theorems, and do not provide an indication on how to design a good trellis source code. Stewart, Gray, and Linde [18], have developed an algorithm for the design of good trellis codes. Simulation results show the algorithm perform better than vector quantizer with the same complexity [9]. Having the reduction of the computational complexity of trellis source codes in mind, Marcellin and Fischer [14] have suggested a new trellis source coding technique called trellis coded quantization (TCQ). TCQ is a form of trellis source coding that was motivated by the analogy to Ungerboeck's [19] trellis coded modulation (TCM). Their simulation results indicate that TCQ is superior to block source coding in the sense of having better performance/complexity tradeoffs. A generalization of TCQ to the vector case (referred to as TCVQ) was presented in [6] and [24]. Fischer and Wang [5], Marcellin [15], and Yang and Zhang [26], combined entropy coding with TCQ to obtain further improvement. An analytical proof for the superiority of trellis source codes over block source codes in terms of performance/complexity, was provided by Zhou and Zhang [29]. They showed, for memoryless sources, that for the same computational complexity $C_{c}$, the redundancy (the difference between the code distortion and the distortion-rate function) for 
a trellis source code is smaller than the redundancy of a block source code [28] by a factor of roughly $\ln \ln C_{c}$. Yang and Zhang [27], further showed that a universal trellis source code has a redundancy which is at the same order as the redundancy of a trellis source code with known statistics [29].

Motivated by the performance/complexity tradeoff superiority of trellis source codes over block source codes, we investigate the error exponent for trellis source coding. By employing Viterbi and Omura's "forbidden" trellis technique [21], we develop a single-letter lower bound on the trellis source coding error exponent. For the same computational complexity, we compare this bound with the error exponent of block source coding previously obtained by Marton [16]. In that case, the bound on the trellis error exponent is given by $\frac{1}{c} R(R-R(D))$, where $c$ is a constant depending on the source alphabet's cardinality, $R$ is the code rate, and $R(D)$ is the source rate-distortion function. For the binary source with Hamming distortion measure, and for rates close to the rate-distortion curve, $R=(1+\varepsilon) R(D), \varepsilon<<1$, we show that the ratio between the trellis error exponent and the block error exponent is lower bounded by a term of the order $O\left(\frac{1}{\varepsilon}\right)$. This means that the ratio between the trellis error exponent and the block error exponent grows without bound as $\varepsilon \rightarrow 0$. This is yet another indication on the superiority of trellis source codes over block source codes.

The outline of the paper is as follows. In Section 2, we review the problem of trellis source coding and introduce the notation. In Section 3, we derive the lower bound on the trellis source coding error exponent. In Section 4, we compare the bound on the trellis exponent with Marton's exponent and calculate the ratio between the two exponents for rates close to the rate-distortion curve. Section 5 contains some concluding remarks and directions for future research.

\section{Problem Formulation}

We begin by setting up the notation. Random variables are denoted by capital letters, their realizations by the corresponding lower case letters, and their alphabets by script letters. 
Similarly, random vectors, their realizations, and their alphabets are denoted, respectively, by boldface capital letters, the corresponding boldface lower case letters and by script letters superscripted by the vector dimension. A substring of vector $\boldsymbol{x}=\left(x_{1}, \ldots, x_{n}\right)$ is written as $x_{i}^{j}=\left(x_{i}, \ldots, x_{j}\right), i \leq j$. Let $\mathcal{X}$ be a finite set. The cardinality of $\mathcal{X}$ is denoted by $|\mathcal{X}|$. We denote a probability mass function (pmf) of random variable $X$ by a capital letter subscripted by $X$, e.g., $P_{X}=\left\{P_{X}(x), x \in \mathcal{X}\right\}$. Similarly, a conditional pmf from a random variable $X \in \mathcal{X}$ to another random variable $Y \in \mathcal{Y}$ is denoted by $P_{Y \mid X}=$ $\left\{P_{Y \mid X}(y \mid x),(x, y) \in \mathcal{X} \times \mathcal{Y}\right\}$. For a given pmf $P_{X}$, the joint pmf of the random variables $X, Y$ induced by the conditional pmf $P_{Y \mid X}$, is denoted by $P_{X Y}=\left\{P_{X}(x) P_{Y \mid X}(y \mid x),(x, y) \in\right.$ $\mathcal{X} \times \mathcal{Y}\}$. The rate-distortion function of a discrete memoryless source (DMS) with a general pmf $Q_{X}$ is denoted by $R\left(Q_{X}, D\right)$. But when $Q_{X}$ is the pmf of the underlying source, it will sometimes be denoted simply by $R(D)$.

Next, we describe the problem of trellis source coding. The considered trellis source coding scheme is described in Fig 1 . We assume a DMS $\left\{X_{i}\right\}$ with a finite alphabet $\mathcal{X}$, and pmf $P_{X}$. The encoder, $g: \mathcal{X}^{n L} \rightarrow\{0, \ldots, q-1\}^{L}$, encodes a sequence of $n L$ source symbols, $\boldsymbol{x} \in \mathcal{X}^{n L}$, into a codeword $\boldsymbol{w}$ of length $L$ with components chosen from the alphabet $\{0, \ldots, q-1\}, \boldsymbol{w} \in\{0, \ldots, q-1\}^{L}$. The coding rate is defined as:

$$
R=\frac{\ln q}{n}\left[\frac{\text { nats }}{\text { symbol }}\right] .
$$

The decoder, $\mathcal{F}_{L}$, time-varying in general, consists of a sequence of $L$ functions (one function for each time instant),

$$
\mathcal{F}_{L} \triangleq\left\{f_{i}:\{0, \ldots, q-1\}^{K} \rightarrow \mathcal{Y}^{n}, \quad i=0,1, \ldots, L-1\right\}
$$

where $K$ is the constraint length, and $\mathcal{Y}$ is the reproduction alphabet assumed to be finite. For a given codeword $\boldsymbol{w} \in\{0, \ldots, q-1\}^{L}$ the decoder's output at time instant $i$ is a vector of length $n, \boldsymbol{y}_{i n}^{(i+1) n-1}=f_{i}\left(\boldsymbol{w}_{i-K+1}^{i}\right), i=0,1, \ldots, L-1$. Let

$$
\alpha \triangleq \frac{K}{L}<1 .
$$


We consider an additive distortion measure

$$
d(\boldsymbol{x}, \boldsymbol{y})=\frac{1}{n L} \sum_{i=0}^{n L-1} d\left(x_{i}, y_{i}\right), \quad(\boldsymbol{x}, \boldsymbol{y}) \in \mathcal{X}^{n L} \times \mathcal{Y}^{n L},
$$

where $d(x, y)$ is bounded distortion measure,

$$
d(x, y) \leq d_{\max }<\infty, \quad \forall(x, y) \in \mathcal{X} \times \mathcal{Y}
$$

Let $\boldsymbol{x}_{i} \triangleq \boldsymbol{x}_{i n}^{(i+1) n-1}, \boldsymbol{y}_{i} \triangleq \boldsymbol{y}_{i n}^{(i+1) n-1}$, and define

$$
d_{n}\left(\boldsymbol{x}_{i}, \boldsymbol{y}_{i}\right) \triangleq \frac{1}{n} \sum_{j=0}^{n-1} d\left(x_{n i+j}, y_{n i+j}\right)
$$

then,

$$
d(\boldsymbol{x}, \boldsymbol{y})=\frac{1}{L} \sum_{i=0}^{L-1} d_{n}\left(\boldsymbol{x}_{i}, \boldsymbol{y}_{i}\right) .
$$

For a given decoder $\mathcal{F}_{L}$, the optimal encoder $g$ is clearly given by

$$
g\left(\boldsymbol{x}, \mathcal{F}_{L}\right)=\arg \min _{\boldsymbol{w} \in\{0,1, \ldots, q-1\}^{L}} d\left(\boldsymbol{x}, \boldsymbol{y}\left(\boldsymbol{w}, \mathcal{F}_{L}\right)\right)
$$

which is practically implemented using trellis search algorithm such as the Viterbi algorithm [22]. Let $\boldsymbol{w}^{*}$ and $\boldsymbol{y}^{*}\left(\boldsymbol{x}, \mathcal{F}_{L}\right)$ be the minimum distortion path sequence and the corresponding decoder output respectively. Let $\left\{\mathcal{F}_{L}\right\}$ denote a sequence of decoders $\left\{\mathcal{F}_{1}, \mathcal{F}_{2}, \mathcal{F}_{3}, \ldots\right\}$. We are interested in evaluating the error exponent,

$$
E_{T}\left(R, D, \alpha,\left\{\mathcal{F}_{L}\right\}\right) \triangleq \liminf _{L \rightarrow \infty}-\frac{1}{n L} \ln \operatorname{Pr}\left\{d\left(\boldsymbol{X}^{*}, \boldsymbol{Y}^{*}\left(\boldsymbol{X}, \mathcal{F}_{L}\right)\right)>D\right\}
$$

where $D>0$ is some given distortion level, when averaging over an ensemble (described next) of $\left\{\mathcal{F}_{L}\right\}$.

\section{Lower Bound on the Error Exponent}

In the following theorem, by averaging over an ensemble of decoders sequences, we obtain a lower bound on (9). Clearly, this bound must also hold for at least one specific sequence of decoders. 


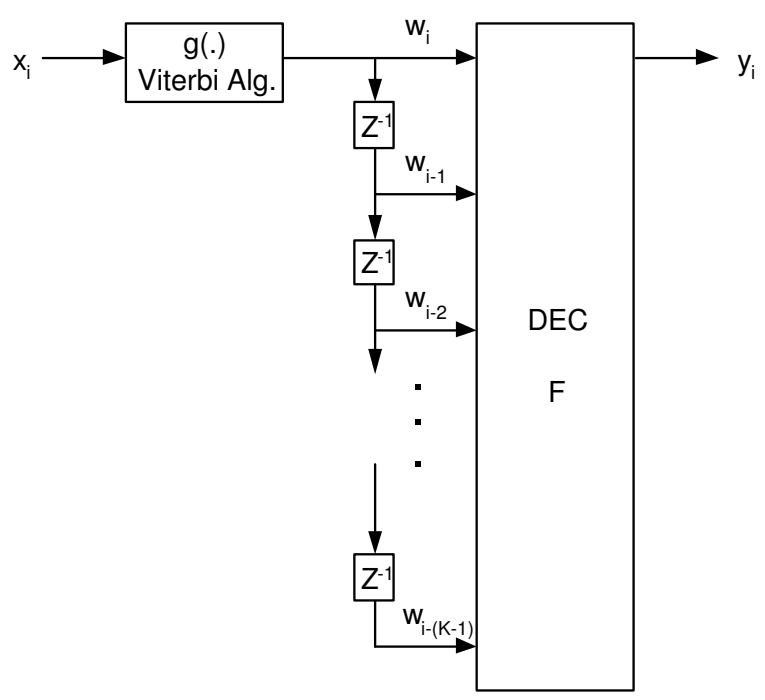

Figure 1: Trellis source coding scheme.

Theorem 3.1. For every conditional pmf

$$
P_{Y \mid X} \in \mathcal{P}(R, D) \triangleq\left\{P_{Y \mid X}: R>I(X ; Y), D>E d(X, Y)\right\},
$$

there exists a sequence of decoders $\left\{\mathcal{F}_{L}\right\}$ that satisfies

$$
E_{T}\left(R, D, \alpha,\left\{\mathcal{F}_{L}\right\}\right) \geq \bar{E}_{T}\left(R, D, \alpha, P_{Y \mid X}\right)>0
$$

where,

$$
\bar{E}_{T}\left(R, D, \alpha, P_{Y \mid X}\right) \triangleq \min \left\{\inf _{\left\{Q_{X Y}: E d(X, Y)=D\right\}} D\left(Q_{X Y} \| P_{X Y}\right), \frac{\alpha}{c} R(R-I(X ; Y))\right\},
$$

$Q_{X Y}$ is an arbitrary joint pmf, $P_{X Y}$ is the joint pmf induced by $P_{Y \mid X}, D\left(Q_{X Y} \| P_{X Y}\right)$ is the relative entropy between $Q_{X Y}$ and $P_{X Y}, I(X ; Y)$ is the mutual information with respect to the joint pmf $P_{X Y}$, and $c \triangleq 2+16(\ln |\mathcal{X}|)^{2}$.

Proof. We use the following random coding argument due to Viterbi and Omura [21]. For an arbitrarily chosen conditional pmf $P_{Y \mid X}$ :

1. Randomly select the decoder $\mathcal{F}_{L}$ by independently selecting the vectors $f_{i}\left(\boldsymbol{x}_{i-K+1}^{i}\right)$, $\boldsymbol{x}_{i-K+1}^{i} \in q^{K}, i=0,1, \ldots, L-1$, according to the pmf

$$
P_{\boldsymbol{Y}}\left(\boldsymbol{y}_{i}\right)=\prod_{j=0}^{n-1} P_{Y}\left(y_{i j}\right)
$$


where $y_{i j}$ is the $j$-th component of the vector $\boldsymbol{y}_{i}$ and,

$$
P_{Y}(y)=\sum_{x \in \mathcal{X}} P_{X}(x) P_{Y \mid X}(y \mid x)
$$

(essentially, independent random selection of $n L q^{K}$ variables according to $P_{Y}(y)$ ).

2. For the selected decoder $\mathcal{F}_{L}$ and a given source sequence, $\boldsymbol{x}$, replace the branch outputs of the all-zero state path (represented by $\left.f_{i}(\mathbf{0}), i=0,1, \ldots, L-1\right)$ of the trellis by an output sequence, $\boldsymbol{y}_{0}$, which is randomly selected according to the density

$$
P_{\boldsymbol{Y}_{0} \mid \boldsymbol{X}}\left(\boldsymbol{y}_{0} \mid \boldsymbol{x}\right)=\prod_{j=0}^{n L-1} P_{Y \mid X}\left(y_{0 j} \mid x_{j}\right) .
$$

The trellis diagram with the replaced all-zero state path is referred to as the "forbidden" trellis diagram. Let $\boldsymbol{y}^{\prime \prime}=\boldsymbol{y}^{\prime \prime}\left(\boldsymbol{x}, \mathcal{F}_{L}, \boldsymbol{y}_{0}\right)$ be the output of the minimum distortion path of the forbidden trellis diagram. $\boldsymbol{y}^{\prime \prime}$ defines a path through the forbidden trellis diagram. Let $\boldsymbol{y}^{\prime}=\boldsymbol{y}^{\prime}\left(\boldsymbol{x}, \mathcal{F}_{L}, \boldsymbol{y}_{0}\right)$ be the output of the corresponding path in the original trellis diagram. $\boldsymbol{y}^{\prime}$ and $\boldsymbol{y}^{\prime \prime}$ are the same except for the outputs on the branches of the all-zero state path. From the definition of $\boldsymbol{y}^{*}\left(\boldsymbol{x}, \mathcal{F}_{L}\right)$ we have that

$$
d\left(\boldsymbol{x}, \boldsymbol{y}^{*}\left(\boldsymbol{x}, \mathcal{F}_{L}\right)\right) \leq d\left(\boldsymbol{x}, \boldsymbol{y}^{\prime}\left(\boldsymbol{x}, \mathcal{F}_{L}, \boldsymbol{y}_{0}\right)\right)
$$

Let

$\mathcal{I} \triangleq\left\{i: \boldsymbol{y}_{i}^{\prime}\left(\boldsymbol{x}, \mathcal{F}_{L}, \boldsymbol{y}_{0}\right)\right.$ is a branch output vector of the all-zero state path $\}$ 
Then,

$$
\begin{aligned}
d\left(\boldsymbol{x}, \boldsymbol{y}^{\prime}\left(\boldsymbol{x}, \mathcal{F}_{L}, \boldsymbol{y}_{0}\right)\right) & =\frac{1}{L} \sum_{i=0}^{L-1} d_{n}\left(\boldsymbol{x}_{i}, \boldsymbol{y}_{i}^{\prime}\left(\boldsymbol{x}, \mathcal{F}_{L}, \boldsymbol{y}_{0}\right)\right) \\
& =\frac{1}{L} \sum_{i \notin \mathcal{I}} d_{n}\left(\boldsymbol{x}_{i}, \boldsymbol{y}_{i}^{\prime \prime}\left(\boldsymbol{x}, \mathcal{F}_{L}, \boldsymbol{y}_{0}\right)\right)+\frac{1}{L} \sum_{i \in \mathcal{I}} d_{n}\left(\boldsymbol{x}_{i}, \boldsymbol{y}_{i}^{\prime}\left(\boldsymbol{x}, \mathcal{F}_{L}, \boldsymbol{y}_{0}\right)\right) \\
& \leq \frac{1}{L} \sum_{i=0}^{L-1} d_{n}\left(\boldsymbol{x}_{i}, \boldsymbol{y}_{i}^{\prime \prime}\left(\boldsymbol{x}, \mathcal{F}_{L}, \boldsymbol{y}_{0}\right)\right)+\frac{1}{L} \sum_{i \in \mathcal{I}} d_{n}\left(\boldsymbol{x}_{i}, \boldsymbol{y}_{i}^{\prime}\left(\boldsymbol{x}, \mathcal{F}_{L}, \boldsymbol{y}_{0}\right)\right) \\
& \leq \frac{1}{L} \sum_{i=0}^{L-1} d_{n}\left(\boldsymbol{x}_{i}, \boldsymbol{y}_{0 i}\right)+\frac{1}{L} \sum_{i \in \mathcal{I}} d_{n}\left(\boldsymbol{x}_{i}, \boldsymbol{y}_{i}^{\prime}\left(\boldsymbol{x}, \mathcal{F}_{L}, \boldsymbol{y}_{0}\right)\right) \\
& =d\left(\boldsymbol{x}, \boldsymbol{y}_{0}\right)+\frac{1}{L} \sum_{i \in \mathcal{I}} d_{n}\left(\boldsymbol{x}_{i}, \boldsymbol{y}_{i}^{\prime}\left(\boldsymbol{x}, \mathcal{F}_{L}, \boldsymbol{y}_{0}\right)\right) \\
& \leq d\left(\boldsymbol{x}, \boldsymbol{y}_{0}\right)+\frac{d_{\max }}{L}|\mathcal{I}|
\end{aligned}
$$

where $|\mathcal{I}|$ is the cardinality of $\mathcal{I}$. Substituting (23) into (16), we have

$$
d\left(\boldsymbol{x}, \boldsymbol{y}^{*}\left(\boldsymbol{x}, \mathcal{F}_{L}\right)\right) \leq d\left(\boldsymbol{x}, \boldsymbol{y}_{0}\right)+\frac{d_{\max }}{L}|\mathcal{I}|
$$

We show that the bound in (11) holds when averaging on $\left\{\mathcal{F}_{L}\right\}$, thus there exists $\left\{\mathcal{F}_{L}\right\}$ for which the bound holds. Averaging over $\mathcal{F}_{L}$, we have

$$
E_{\mathcal{F}_{L}} \operatorname{Pr}\left\{d\left(\boldsymbol{X}, \boldsymbol{Y}^{*}\left(\boldsymbol{X}, \mathcal{F}_{L}\right)\right)>D \mid \mathcal{F}_{L}\right\}=\operatorname{Pr}\left\{d\left(\boldsymbol{X}, \boldsymbol{Y}^{*}\left(\boldsymbol{X}, \mathcal{F}_{L}\right)\right)>D\right\}
$$

From (24), we have

$$
\begin{aligned}
\operatorname{Pr}\left\{d\left(\boldsymbol{X}, \boldsymbol{Y}^{*}\left(\boldsymbol{X}, \mathcal{F}_{L}\right)\right)>D\right\} & \leq \operatorname{Pr}\left\{d\left(\boldsymbol{X}, \boldsymbol{Y}_{0}\right)+\frac{d_{\text {max }}}{L}|\mathcal{I}|>D\right\} \\
& =\operatorname{Pr}\left\{d\left(\boldsymbol{X}, \boldsymbol{Y}_{0}\right)+\frac{d_{\text {max }}}{L}|\mathcal{I}|>D, \frac{d_{\text {max }}}{L}|\mathcal{I}| \geq \varepsilon\right\} \\
& +\operatorname{Pr}\left\{d\left(\boldsymbol{X}, \boldsymbol{Y}_{0}\right)+\frac{d_{\text {max }}}{L}|\mathcal{I}|>D, \frac{d_{\text {max }}}{L}|\mathcal{I}|<\varepsilon\right\}
\end{aligned}
$$

for every $\varepsilon$. Since

$$
\begin{aligned}
\operatorname{Pr}\left\{d\left(\boldsymbol{X}, \boldsymbol{Y}_{0}\right)+\frac{d_{\text {max }}}{L}|\mathcal{I}|>D, \frac{d_{\text {max }}}{L}|\mathcal{I}|<\varepsilon\right\} & \leq \operatorname{Pr}\left\{d\left(\boldsymbol{X}, \boldsymbol{Y}_{0}\right)>D-\varepsilon, \frac{d_{\text {max }}}{L}|\mathcal{I}|<\varepsilon\right\} \\
& \leq \operatorname{Pr}\left\{d\left(\boldsymbol{X}, \boldsymbol{Y}_{0}\right)>D-\varepsilon\right\}
\end{aligned}
$$

and since

$$
\operatorname{Pr}\left\{d\left(\boldsymbol{X}, \boldsymbol{Y}_{0}\right)+\frac{d_{\text {max }}}{L}|\mathcal{I}|>D, \frac{d_{\text {max }}}{L}|\mathcal{I}| \geq \varepsilon\right\} \leq \operatorname{Pr}\left\{\frac{d_{\text {max }}}{L}|\mathcal{I}| \geq \varepsilon\right\},
$$


we obtain

$$
\operatorname{Pr}\left\{d\left(\boldsymbol{X}, \boldsymbol{Y}^{*}\left(\boldsymbol{X}, \mathcal{F}_{L}\right)\right)>D\right\} \leq \operatorname{Pr}\left\{d\left(\boldsymbol{X}, \boldsymbol{Y}_{0}\right)>D-\varepsilon\right\}+\operatorname{Pr}\left\{\frac{d_{\max }}{L}|\mathcal{I}| \geq \varepsilon\right\}
$$

As for the first term of (29), using Chernoff bound [3] we get,

$$
\begin{aligned}
\operatorname{Pr}\left\{d\left(\boldsymbol{X}, \boldsymbol{Y}_{0}\right)>D-\varepsilon\right\} & =\operatorname{Pr}\left\{\sum_{i=0}^{n L-1} d\left(X_{i}, Y_{0 i}\right)>n L(D-\varepsilon)\right\} \\
& \leq \inf _{s \geq 0}\left\{\exp \{-s n L(D-\varepsilon)\} E \exp \left\{s \sum_{i=0}^{n L-1} d\left(X_{i}, Y_{0 i}\right)\right\}\right\} \\
& =\inf _{s \geq 0}\left\{\exp \{-\operatorname{sn} L(D-\varepsilon)\}(E \exp \{s d(X, Y)\})^{n L}\right\} \\
& =\exp \left\{-n L \sup _{s \geq 0}[s(D-\varepsilon)-\ln (E \exp (s d(X, Y)))]\right\}
\end{aligned}
$$

Equation (30) is due to the fact that $\left\{\left(X_{i}, Y_{0 i}\right)\right\}$ are i.i.d. Using Jensen's inequality [4] one can obtain that

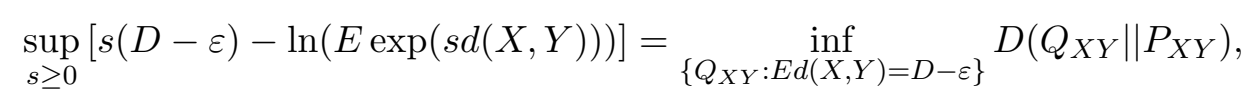

resulting in that,

$$
\operatorname{Pr}\left\{d\left(\boldsymbol{X}, \boldsymbol{Y}_{0}\right)>D-\varepsilon\right\} \leq \exp \left\{-n L \inf _{\left\{Q_{X Y}: E d(X, Y)=D-\varepsilon\right\}} D\left(Q_{X Y} \| P_{X Y}\right)\right\} .
$$

Note that

$$
\inf _{\left\{Q_{X Y}: E d(X, Y)=D-\varepsilon\right\}} D\left(Q_{X Y} \| P_{X Y}\right)>0
$$

for

$$
D-\varepsilon>E d(X, Y)
$$

As for the second term of (29), taking $\varepsilon>0$ and using Markov's inequality, we have

$$
P_{r}\left\{\frac{d_{\max }}{L}|\mathcal{I}| \geq \varepsilon\right\} \leq \frac{d_{\max }}{\varepsilon L} E|\mathcal{I}|
$$

Let $p_{j i}$ be the probability that $\boldsymbol{y}^{\prime}$ merges with the all-zero state path at node $j$ and remains merged for exactly $i$ branches. Using the results of Viterbi and Omura [21], we have

$$
E|\mathcal{I}| \leq \sum_{j=0}^{L-1} \sum_{i=1}^{L-j-1} i p_{j i}
$$


where,

$$
\begin{gathered}
p_{j i} \leq \exp \left\{-(K-1) E\left(R, P_{Y \mid X}\right) \ln q\right\} \exp \left\{-i \frac{n E^{2}\left(R, P_{Y \mid X}\right) c}{2}\right\} \\
E\left(R, P_{Y \mid X}\right) \triangleq \frac{R-I(X ; Y)}{c}
\end{gathered}
$$

Substituting (38) into (37), we get

$$
\begin{aligned}
E|\mathcal{I}| & \leq \exp \left\{-(K-1) E\left(R, P_{Y \mid X}\right) \ln q\right\} \sum_{j=0}^{L-1} \sum_{i=1}^{L-j-1} i \exp \left\{-i \frac{n E^{2}\left(R, P_{Y \mid X}\right) c}{2}\right\} \\
& \leq \exp \left\{-(K-1) E\left(R, P_{Y \mid X}\right) \ln q\right\} L \sum_{i=1}^{L-1} i \exp \left\{-i \frac{n E^{2}\left(R, P_{Y \mid X}\right) c}{2}\right\} \\
& \leq \exp \left\{-(K-1) E\left(R, P_{Y \mid X}\right) \ln q\right\} L \sum_{i=1}^{\infty} i \exp \left\{-i \frac{n E^{2}\left(R, P_{Y \mid X}\right) c}{2}\right\} \\
& =L \frac{\exp \left\{-\frac{n E^{2}\left(R, P_{Y \mid X}\right) c}{2}\right\}}{\left(1-\exp \left\{-\frac{n E^{2}\left(R, P_{Y \mid X}\right) c}{2}\right\}\right)^{2}} \exp \left\{-(K-1) E\left(R, P_{Y \mid X}\right) \ln q\right\} \\
& =L \frac{\exp \left\{-\frac{n E^{2}\left(R, P_{Y \mid X}\right) c}{2}+E\left(R, P_{Y \mid X}\right) \ln q\right\}}{\left(1-\exp \left\{-\frac{n E^{2}\left(R, P_{Y \mid X}\right) c}{2}\right\}\right)^{2} \exp \left\{-K E\left(R, P_{Y \mid X}\right) \ln q\right\} .}
\end{aligned}
$$

Equation (40) holds for $R-I(X ; Y)>0$, otherwise the sum does not converge. Let

$$
\psi(\varepsilon) \triangleq \frac{d_{\max }}{\varepsilon} \frac{\exp \left\{-\frac{n E^{2}\left(R, P_{Y \mid X}\right) c}{2}+E\left(R, P_{Y \mid X}\right) \ln q\right\}}{\left(1-\exp \left\{-\frac{n E^{2}\left(R, P_{Y \mid X}\right) c}{2}\right\}\right)^{2}},
$$

then, substituting (41) into (36), we obtain

$$
\begin{aligned}
\operatorname{Pr}\left\{\frac{d_{\text {max }}}{L}|\mathcal{I}| \geq \varepsilon\right\} & \leq \psi(\varepsilon) \exp \left\{-L \alpha E\left(R, P_{Y \mid X}\right) \ln q\right\} \\
& =\psi(\varepsilon) \exp \left\{-n L \alpha E\left(R, P_{Y \mid X}\right) R\right\} .
\end{aligned}
$$

Substituting (33) and (43) into (29), we obtain

$$
\operatorname{Pr}\left\{d\left(\boldsymbol{X}, \boldsymbol{Y}^{*}\left(\boldsymbol{X}, \mathcal{F}_{L}\right)\right)>D\right\} \leq 2 \max [1, \psi(\varepsilon)] \exp \left\{-n L E^{\prime}\left(R, D, \alpha, P_{Y \mid X}, \varepsilon\right)\right\},
$$

where

$$
E^{\prime}\left(R, D, \alpha, P_{Y \mid X}, \varepsilon\right) \triangleq \min \left\{\inf _{\left\{Q_{X Y}: E d(X, Y)=D-\varepsilon\right\}} D\left(Q_{X Y} \| P_{X Y}\right), \frac{\alpha}{c} R(R-I(X ; Y))\right\}
$$


Taking $\varepsilon>0$ to be a function of $L, \varepsilon=\varepsilon(L)$, satisfying

$$
\lim _{L \rightarrow \infty} \varepsilon(L)=0
$$

and

$$
\lim _{L \rightarrow \infty} \frac{1}{L} \ln \varepsilon(L)=0
$$

(e.g., $\varepsilon(L)=1 / L)$, we obtain that,

$$
E_{T}\left(R, D, \alpha,\left\{\mathcal{F}_{L}\right\}\right) \geq \bar{E}_{T}\left(R, D, \alpha, P_{Y \mid X}\right)
$$

From (40) and (34) we have that,

$$
\bar{E}\left(R, D, \alpha, P_{Y \mid X}\right)>0
$$

for

$$
D>E d(X, Y) \text { and } R>I(X ; Y) .
$$

This concludes the proof

Theorem 3.1 actually tells that we can get arbitrarily close to the rate-distortion curve while maintaining a positive error exponent. This can be easily verified by choosing $P_{Y \mid X}$ to be the pmf that achieves the rate-distortion function. However, this pmf is not necessarily the one that achieves the maximal error exponent, given by

$$
\sup _{P_{Y \mid X} \in \mathcal{P}(R, D)} \bar{E}_{T}\left(R, D, \alpha, P_{Y \mid X}\right) .
$$

\section{Example - Binary source with Hamming distortion measure}

Let $\mathcal{X}=\mathcal{Y}=\{0,1\}, P_{X}(1)=p<0.5$, and $d(x, y)=1-\delta(x-y)$. Let us define the random variable $A$ as

$$
A= \begin{cases}1 & X \neq Y \\ 0 & X=Y\end{cases}
$$


Let $Q_{A}$ and $P_{A}$ be the pmf's of $A$ when $(X, Y)$ are distributed according to $Q_{X Y}$ and $P_{X Y}$ respectively, i.e.,

$$
\begin{aligned}
& Q_{A}(1)=Q_{X Y}(0,1)+Q_{X Y}(1,0) \\
& P_{A}(1)=p P_{Y \mid X}(0 \mid 1)+(1-p) P_{Y \mid X}(1 \mid 0)=E d(X, Y) .
\end{aligned}
$$

Using the data processing theorem for relative entropy we have

$$
D\left(Q_{X Y} \| P_{X Y}\right) \geq D\left(Q_{A} \| P_{A}\right)
$$

and thus clearly

$$
\begin{aligned}
\min _{\left\{Q_{X Y}: E d(X, Y)=D\right\}} D\left(Q_{X Y} \| P_{X Y}\right) & =\min _{\left\{Q_{A}: Q_{A}(1)=D\right\}} D\left(Q_{A} \| P_{A}\right) \\
& =D \ln \frac{D}{E d(X, Y)}+(1-D) \ln \frac{1-D}{1-E d(X, Y)} \\
& =-h_{2}(D)-D \ln (E d(X, Y))-(1-D) \ln (1-E d(X, Y)),
\end{aligned}
$$

where $h_{2}(D) \triangleq-D \ln D-(1-D) \ln (1-D)$ is the binary entropy function. Substituting (56) and $c=2+16(\ln 2)^{2}=9.6872$ into (51) we obtain that,

$$
\begin{aligned}
E_{T}\left(R, D, \alpha,\left\{\mathcal{F}_{L}\right\}\right) \geq \sup _{\left\{P_{Y \mid X}: R>I(X ; Y), D>E d(X, Y)\right\}} \min \{ & -h_{2}(D)-D \ln (E d(X, Y)) \\
& -(1-D) \ln (1-E d(X, Y)), \\
& 0.1032 \alpha R(R-I(X ; Y))\},
\end{aligned}
$$

which can be easily evaluated numerically.

\section{Comparison with the Error Exponent for Block Source Coding}

We would like to compare the lower bound on the trellis error exponent with the error exponent corresponding to block source codes for the same computational complexity. Marton 
[16] has shown that the best block code satisfies,

$$
\begin{aligned}
E_{B}(R, D) & \triangleq \lim _{N \rightarrow \infty}-\frac{1}{N} \ln \operatorname{Pr}\{d(\boldsymbol{X}, \boldsymbol{Y})>D\} \\
& =\min _{\left\{Q_{X}: R\left(Q_{X}, D\right) \geq R\right\}} D\left(Q_{X} \| P_{X}\right),
\end{aligned}
$$

where the code rate satisfy $R(D)<R<\max _{Q_{X}} R\left(Q_{X}, D\right)$. For block source codes, the encoder computational complexity grows exponentially fast with exponent of $N R$, while for trellis source codes it grows exponentially fast with exponent of $\alpha n L R$. For the same computational complexity exponent ${ }^{1}$

$$
\alpha n L R=N R,
$$

the error exponent for block source codes is given by $E_{B}(R, D)$, while for trellis source codes it is given by

$$
\frac{1}{\alpha} E_{T}\left(R, D, \alpha,\left\{\mathcal{F}_{L}\right\}\right) \geq \frac{1}{\alpha} \sup _{P_{Y \mid X} \in \mathcal{P}(R, D)} \bar{E}_{T}\left(R, D, \alpha, P_{Y \mid X}\right) .
$$

Striving for the highest exponent we define,

$$
E_{T}\left(R, D,\left\{\mathcal{F}_{L}\right\}\right) \triangleq \sup _{0<\alpha<1} \frac{1}{\alpha} E_{T}\left(R, D, \alpha,\left\{\mathcal{F}_{L}\right\}\right)
$$

Using (60) we have that,

$$
\begin{aligned}
E_{T}\left(R, D,\left\{\mathcal{F}_{L}\right\}\right) & \geq \sup _{0<\alpha<1} \sup _{P_{Y \mid X} \in \mathcal{P}(R, D)} \frac{1}{\alpha} \bar{E}_{T}\left(R, D, \alpha, P_{Y \mid X}\right) \\
& =\sup _{0<\alpha<1} \sup _{P_{Y \mid X} \in \mathcal{P}(R, D)} \min \left\{\frac{1}{\alpha} \inf _{\left\{Q_{X Y}: E d(X, Y)=D\right\}} D\left(Q_{X Y} \| P_{X Y}\right), \frac{1}{c} R(R-I(X ; Y))\right\} \\
& =\sup _{P_{Y \mid X} \in \mathcal{P}(R, D)} \frac{1}{c} R(R-I(X ; Y)) \\
& =\frac{1}{c} R\left(R-\inf _{P_{Y \mid X} \in \mathcal{P}(R, D)} I(X ; Y)\right) \\
& =\frac{1}{c} R(R-R(D))
\end{aligned}
$$

\footnotetext{
${ }^{1}$ Note that the trellis codeword length is actually $L+K-1$. This is because the codeword must be ended by a tail of $K-1$ zeros that reset the decoder memory. At first look one might think that this leads to a reduction in the coding rate. However, the code rate being defined as $\ln \left(\operatorname{size}\right.$ of codebook) $\left(\ln q^{L}\right)$ divided by the length of the source input sequence $(n L)$, there is actually no rate loss. When comparing between the block code and trellis code, the penalty of using an additional $K-1$ channel uses in the trellis code does not matter since anyway we have that $L=\frac{N}{n \alpha}>>N$.
} 
for $R>R(D)$. The trellis exponent is superior to the block exponent if

$$
\frac{E_{T}\left(R, D,\left\{\mathcal{F}_{L}\right\}\right)}{E_{B}(R, D)} \geq \frac{R(R-R(D))}{c \min _{\left\{Q_{X}: R\left(Q_{X}, D\right) \geq R\right\}} D\left(Q_{X} \| P_{X}\right)}>1
$$

We now return to the binary example. Marton has shown [16] that

$$
E_{B}(R, D)=q \ln \frac{q}{p}+(1-q) \ln \frac{1-q}{1-p}
$$

where $q<0.5$ is the solution to the equation,

$$
h_{2}(q)=h_{2}(D)+R
$$

where,

$$
h_{2}(p)-h_{2}(D)=R(D)<R<\ln 2-h_{2}(D), \quad D \leq p .
$$

Without generality loss, assume that

$$
R=(1+\varepsilon) R(D),
$$

where

$$
0<\varepsilon<\frac{\ln 2-h_{2}(D)}{h_{2}(p)-h_{2}(D)}-1
$$

We now derive an explicit expression for the lower bound on $E_{T}\left(R, D,\left\{\mathcal{F}_{L}\right\}\right) / E_{B}(R, D)$ (63). Substituting (67) into (65), we have

$$
h_{2}(q)=h_{2}(p)+\varepsilon R(D) .
$$

Let $q(\varepsilon, p, D)<0.5$ denote the $q$ that solves equation (69). Using Lagrange's mid-value theorem we have

$$
q(\varepsilon, p, D)-p=\frac{h_{2}(q(\varepsilon, p, D))-h_{2}(p)}{\left.\frac{d h_{2}(x)}{d x}\right|_{x=\xi(\varepsilon, p, D)}}=\frac{\varepsilon R(D)}{\ln \left(\frac{1}{\xi(\varepsilon, p, D)}-1\right)},
$$

where $\xi(\varepsilon, p, D)$ is a point satisfying

$$
p<\xi(\varepsilon, p, D)<q(\varepsilon, p, D)<0.5 \text {. }
$$

Note that

$$
0<\ln \left(\frac{1}{q(\varepsilon, p, D)}-1\right)<\ln \left(\frac{1}{\xi(\varepsilon, p, D)}-1\right)<\ln \left(\frac{1}{p}-1\right)
$$


Substituting (70) into (64) we obtain that

$$
\begin{aligned}
E_{B}(R, D) & =\left(p+\frac{\varepsilon R(D)}{\ln \left(\frac{1}{\xi(\varepsilon, p, D)}-1\right)}\right) \ln \left(1+\frac{\varepsilon R(D)}{p \ln \left(\frac{1}{\xi(\varepsilon, p, D)}-1\right)}\right) \\
& +\left(1-p-\frac{\varepsilon R(D)}{\ln \left(\frac{1}{\xi(\varepsilon, p, D)}-1\right)}\right) \ln \left(1-\frac{\varepsilon R(D)}{(1-p) \ln \left(\frac{1}{\xi(\varepsilon, p, D)}-1\right)}\right) .
\end{aligned}
$$

Using Taylor's series expansion we can write that

$$
(p+x) \ln \left(1+\frac{x}{p}\right)+(1-p-x) \ln \left(1-\frac{x}{1-p}\right)=\frac{1}{2 p(1-p)} x^{2}+\frac{2(p+c)-1}{6(1-(p+c))^{2}(p+c)^{2}} x^{3},
$$

where $c$ is a point satisfying

$$
0<c<x
$$

In our case

$$
x=\frac{\varepsilon R(D)}{\ln \left(\frac{1}{\xi(\varepsilon, p, D)}-1\right)}>0,
$$

and since

$$
p+c<p+x=p+\frac{\varepsilon R(D)}{\ln \left(\frac{1}{\xi(\varepsilon, p, D)}-1\right)}=q(\varepsilon, p, D)<0.5,
$$

we have that the most right-most term in equation (74) is negative, and thus

$$
E_{B}(R, D)<\frac{(\varepsilon R(D))^{2}}{2 p(1-p)\left[\ln \left(\frac{1}{\xi(\varepsilon, p, D)}-1\right)\right]^{2}}
$$

On the other hand, using (62) we have

$$
E_{T}\left(R, D,\left\{\mathcal{F}_{L}\right\}\right) \geq \frac{1}{c} R(R-R(D))=0.1032(1+\varepsilon) \varepsilon(R(D))^{2} .
$$

Substituting (78) and (79) into (63), we obtain that

$$
\frac{E_{T}\left(R, D,\left\{\mathcal{F}_{L}\right\}\right)}{E_{B}(R, D)}>0.2064 p(1-p)\left[\ln \left(\frac{1}{\xi(\varepsilon, p, D)}-1\right)\right]^{2}\left(\frac{1+\varepsilon}{\varepsilon}\right) .
$$

Let us now choose some $\varepsilon_{0}$ satisfying (68), and let $q\left(\varepsilon_{0}, p, D\right)<0.5$ be the solution to equation (69) when $\varepsilon=\varepsilon_{0}$. For $\varepsilon \leq \varepsilon_{0}$, using (72) and since $h_{2}(x)$ is increasing function of $x$ for $x<0.5$, we have that $q(\varepsilon, p, D) \leq q\left(\varepsilon_{0}, p, D\right)<0.5$, and therefore

$$
0<\ln \left(\frac{1}{q\left(\varepsilon_{0}, p, D\right)}-1\right)<\ln \left(\frac{1}{\xi(\varepsilon, p, D)}-1\right)<\ln \left(\frac{1}{p}-1\right) .
$$


Using (81) we obtain that for $\varepsilon \leq \varepsilon_{0}$,

$$
\frac{E_{T}\left(R, D,\left\{\mathcal{F}_{L}\right\}\right)}{E_{B}(R, D)}>O\left(\frac{1}{\varepsilon}\right)
$$

Thus, for small enough $\varepsilon$, i.e, for rates close to $R(D)$, the trellis exponent is superior to the block exponent. Fortunately, keeping in mind we are interested in achieving maximum possible compression, the region where $R$ is very close to $R(D)$ is of the most interest. For $\varepsilon<<1$, we have that $\xi(\varepsilon, p, D) \rightarrow p$, and we can approximate the bound in (80) by

$$
0.2064 p(1-p)\left[\ln \left(\frac{1}{\xi(\varepsilon, p, D)}-1\right)\right]^{2}\left(\frac{1+\varepsilon}{\varepsilon}\right) \approx 0.2064 p(1-p)\left[\ln \left(\frac{1}{p}-1\right)\right]^{2} \frac{1}{\varepsilon}
$$

In Table 1 we have numerically calculated the lower bound on $E_{T}\left(R, D,\left\{\mathcal{F}_{L}\right\}\right) / E_{B}(R, D)$ (63) for $p=0.3$. The number in the brackets is the approximation of the lower bound calculated according to (83). We can see that the approximation is very accurate.

For coding rates that are more remote from $\mathrm{R}(\mathrm{D})$, the lower bound on the trellis exponent was not always found larger than the block exponent. We conjecture that this should be attributed to the possibility that the bound may be loose for these rates, rather than to the possibility that the real trellis exponent is inferior to the block exponent.

\begin{tabular}{|c|c|c|c|}
\hline$\frac{E_{T}\left(R, D,\left\{\mathcal{F}_{L}\right\}\right)}{E_{B}(R, D)}>$ & $R=1.01 R(D)$ & $R=1.001 R(D)$ & $R=1.0001 R(D)$ \\
\hline$D=0.05$ & $3.0669(3.1437)$ & $31.0813(31.1569)$ & $311.2140(311.2895)$ \\
\hline$D=0.10$ & $3.0906(3.1437)$ & $31.1045(31.1569)$ & $311.2372(311.2895)$ \\
\hline$D=0.15$ & $3.1088(3.1437)$ & $31.1224(31.1569)$ & $311.2550(311.2895)$ \\
\hline$D=0.20$ & $3.1232(3.1437)$ & $31.1369(31.1569)$ & $311.2691(311.2895)$ \\
\hline$D=0.25$ & $3.1347(3.1437)$ & $31.1482(31.1569)$ & $311.2805(311.2895)$ \\
\hline
\end{tabular}

Table 1: The ratio between the error exponents for several rate-distortion pairs.

Essentially, we showed that for small $\varepsilon$ the trellis exponent scales linearly with $\varepsilon$, whereas the block exponent is quadratic in $\varepsilon$, so when $\varepsilon$ vanishes, the ratio between them grows without bound. This was done for the binary case which is just an example, but the natural question that arises is whether this behavior applies more generally than in the binary case. 
It is not difficult to show that the answer is affirmative provided that the minimizer of $D\left(Q_{X} \| P_{X}\right)$ s.t. $R\left(Q_{X}, D\right) \geq(1+\varepsilon) R(D)$ is always close to $P_{X}$ (in the variational distance sense) for small $\varepsilon$, because then the divergence will be quadratic in $\varepsilon$, similarly as in the binary example. However, it is not always true that the minimizing $Q_{X}$ is close to $P_{X}$ even if $\varepsilon$ is very small. A problematic case may arise when $R\left(Q_{X}, D\right)$, as a functional of $Q_{X}$ for fixed $D$, has local maxima which are not global maxima [1]. If $P_{X}$ is such a local maximum, then the minimizer $Q_{X}$ of $D\left(Q_{X} \| P_{X}\right)$ might be far away from $P_{X}$ and the above technique does not apply.

For a source $P_{X}$ and a distortion measure which meet the Shannon lower bound (SLB) [2], the above-mentioned local maximum problem does not exist, and so, our analysis for the binary example can essentially be extended. To demonstrate this, consider, for simplicity, the special case where $\mathcal{X}=\mathcal{Y}$ and where $d$ is a difference distortion measure, i.e., $d(x, y)=\rho(x-y)$ for a well-defined subtraction between members of $\mathcal{X}$. Then, the SLB is given by $\left(H\left(Q_{X}\right)\right.$ is the entropy corresponding to $\left.Q_{X}\right)$

$$
R\left(Q_{X}, D\right) \geq H\left(Q_{X}\right)-\phi(D)
$$

where the function $\phi$ is defined as

$$
\phi(D)=\max _{\{Z: E \rho(Z) \leq D\}} H(Z)
$$

$Z$ being a random variable that takes all values of differences between members of $\mathcal{X}$. Now, since we are assuming that the SLB is tight for $P_{X}$, inequality (84) becomes an equality for $Q_{X}=P_{X}$. Thus,

$$
\begin{aligned}
E_{B}(R, D) & =\min _{\left\{Q_{X}: R\left(Q_{X}, D\right) \geq(1+\varepsilon)\left(H\left(P_{X}\right)-\phi(D)\right)\right\}} D\left(Q_{X} \| P_{X}\right) \\
& \leq \min _{\left\{Q_{X}: H\left(Q_{X}\right)-\phi(D) \geq(1+\varepsilon)\left(H\left(P_{X}\right)-\phi(D)\right)\right\}} D\left(Q_{X} \| P_{X}\right) \\
& \leq \min _{\left\{Q_{X}: H\left(Q_{X}\right)-\phi(D)=(1+\varepsilon)\left(H\left(P_{X}\right)-\phi(D)\right)\right\}} D\left(Q_{X} \| P_{X}\right) \\
& =\min _{\left\{Q_{X}: H\left(Q_{X}\right)-H\left(P_{X}\right)=\varepsilon\left(H\left(P_{X}\right)-\phi(D)\right)\right\}} D\left(Q_{X} \| P_{X}\right) .
\end{aligned}
$$

Since $H\left(Q_{X}\right)$ is concave functional of $Q_{X}$ it has only one local maximum which is also the global maximum. For most sources, $H\left(Q_{X}\right)$ being within $\varepsilon$ close to $H\left(P_{X}\right)$, also indicates 
that the distance between $Q_{X}$ and $P_{X}$ is also proportional to $\varepsilon$ (this can be checked for a specific source by calculating the partial derivatives of $H\left(Q_{X}\right)$ for $\left.Q_{X}=P_{X}\right)$. Examples for such sources are all finite alphabet non-uniformly distributed sources with the Hamming distortion measure [2], note however, that for those sources the SLB is usually tight only below some distortion level.

\section{Concluding Remarks and Future Research Directions}

In this paper we developed a lower bound on the trellis source coding error exponent. For binary sources with Hamming distortion measure, for rates close to the rate-distortion curve, and for the same computational complexity, we showed the bound to be superior to the block source coding error exponent. This result can also be extended to sources with distortion measures for which the SLB is tight, e.g., all finite alphabet non-uniformly distributed sources with the Hamming distortion measure and for small values of distortion. An interesting future research direction would be to look into tightness of the trellis error exponent bound. This may be done by exploring more powerful bounding techniques and perhaps also devise upper bounds to the trellis exponent. 


\section{References}

[1] R. Ahlswede, "Extremal properties of rate-distortion functions," IEEE Trans. Information Theory, Vol. 36, no. 1, pp. 166-171, Jan. 1990.

[2] T. Berger, Rate-distortion theory: A mathematical basis for data compression. PrenticeHall, Inc, 1971, pp. 61-63.

[3] T.M. Cover and J.A. Thomas, Elements of Information Theory. John Wiley \& Sons, Inc, 1991, pp. 318, 369.

[4] A. Dembo and O. Zeitouni, Large deviations techniques and applications. Jones \& Bartlett, Inc, 1992, pp. 19,27.

[5] T. R. Fischer and M. Wang, "Entropy-constarined trellis-coded quantization," IEEE Trans. Information Theory, Vol. 38, pp. 415-425, Mar. 1992.

[6] T. R. Fischer, M. W. Marcellin, and M. Wang, "Trellis-coded vector quantization," IEEE Trans. Information Theory, Vol. 37, No. 6, pp. 1551-1566, Nov. 1991.

[7] R. M. Gray, "Sliding-block source coding," IEEE Trans. Information Theory, Vol. 21, No. 4, pp. 357-367, Jul. 1975.

[8] R. M. Gray, "Time-invariant trellis encoding of ergodic discrete-time source with a fidelity criterion," IEEE Trans. Information Theory,Vol. 23, No. 1, pp. 71-83, Jan. 1977.

[9] R. M. Gray, "Vector quantization," IEEE ASSP Magazine,Vol. 1, pp. 4-29, Apr. 1984.

[10] R. M. Gray, D. L. Neuhoff, and D. S. Ornstein, "Nonblock source coding with a fidelity criterion," Ann. Prob, Vol. 3, pp. 478-491, 1975.

[11] R. M. Gray, D. L. Neuhoff, and J. K. Omura, "Process definitions of distortion-rate functions and source coding theorems," IEEE Trans. Information Theory, Vol. 25, pp. 524-532, Sep. 1975. 
[12] R. M. Gray and D. S. Ornstein, "Sliding-block joint source/noisy-channel coding theorems," IEEE Trans. Information Theory, Vol. 22, No. 6, pp. 682-690, Nov. 1976.

[13] F. Jelinek, "Tree encoding of memoryless time-discrete sources with a fidelity criterion," IEEE Trans. Information Theory, Vol. 15, pp. 584-590, Sep. 1969.

[14] M. W. Marcellin and T. R. Fischer, "Trellis coded quantization of memoryless and Gauss-Markov sources," IEEE Trans. Communications, Vol. 38, No. 1, pp. 82-93, Jan. 1990.

[15] M. W. Marcellin, "On entropy constarined TCQ," IEEE Trans. Communications, Vol. 42, pp. 14-16, Jan. 1994.

[16] K. Marton, "Error exponent for source coding with a fidelity criterion," IEEE Trans. Information Theory, Vol. 20, No. 2, pp. 197-199, Mar. 1974.

[17] J. K. Omura, "On the Viterbi algortithm for source coding," in Proc. 1972 IEEE Int. Symp. on Information Theory, Pacific Grove, California, USA, p. 21.

[18] L. C. Stewart, R. M. Gray, and Y. Linde, "The design of trellis waveform coders," IEEE Trans. Communications, Vol. 30, No. 4, pp. 702-710, Apr. 1982.

[19] G. Ungerboeck, "Channel coding with multilevel/phase signals," IEEE Trans. Information Theory, Vol. 28, pp. 55-67, Jan.1982.

[20] A. J. Viterbi, "Error bounds for convolutional codes an asymptotically optimum decoding algorithm," IEEE Trans. Information Theory, Vol. 13, pp. 260-269, Apr. 1967.

[21] A. J. Viterbi and J.K. Omura, "Trellis encoding of memoryless discrete-time sources with a fidelity criterion," IEEE Trans. Information Theory, Vol. 20, No. 3, pp. 325-332, May. 1974.

[22] A. J. Viterbi and J.K. Omura, Principles of Digital Communication and Coding. McGraw-Hill, Inc, 1979, pp. 415-421. 
[23] M. Wang and T. R. Fischer, "Trellis-coded quantization designed for noisy channels," IEEE Trans. Information Theory, Vol. 40, No. 6, pp. 1792-1802, Nov. 1994.

[24] H. S. Wang and N. Moayeri, "Trellis coded vector quantization," IEEE Trans. Communications, Vol. 40, pp. 1273-1276, Aug. 1992.

[25] L. Yang and T. R. Fischer, "A new trellis source code for memoryless sources," IEEE Trans. Information Theory, Vol. 44, pp. 3056-3063, Nov. 1998.

[26] E. H. Yang and Z. Zhang, "Variable-rate trellis source coding," IEEE Trans. Information Theory, Vol. 45, No. 4, pp. 586-608, Mar. 1999.

[27] J. Yang and Z. Zhang, "On the redundancy of universal trellis lossy source coding," in Proc. 2003 IEEE Int. Symp. on Information Theory, Yokahoma, Japan, p. 168.

[28] Z. Zhang, E. H. Yang, and V. K. Wei, "The redundancy of source coding with a fidelity criterion - Part one: Known statistics," IEEE Trans. Information Theory, Vol. 43, pp. 71-91, Jan. 1997.

[29] G. C. Zhou and Z. Zhang, "On the redundancy of trellis lossy source coding," IEEE Trans. Information Theory, Vol. 48, No. 1, pp. 205-218, Jan. 2002. 